\title{
An abundance analysis of slowly pulsating B stars
}

\author{
M. Briquet, ${ }^{1}$ T. Morel ${ }^{1,2}$ \\ ${ }^{1}$ Instituut voor Sterrenkunde, Katholieke Univ. Leuven, Celestijnenlaan 200 D, B-3001 Leuven, Belgium \\ ${ }^{2}$ European Space Agency (ESA) postdoctoral external fellow
}

\begin{abstract}
We present the methodology and the first results of a study aimed to determine in a selfconsistent way the physical parameters $T_{\text {eff }}$ and $\log g$ as well as NLTE abundances of the majority of all confirmed slowly pulsating B stars (hereafter SPBs).
\end{abstract}

\section{Observations}

High-resolution optical spectra of more than $30 \mathrm{SPBs}$ were obtained with the CORALIE echelle spectrograph attached to the 1.2-m Leonard Euler telescope at La Silla during many observing runs dedicated to this class of pulsating B stars. In order to minimize the impact of the pulsations, several time-resolved spectra were co-added.

\section{Methods of analysis}

We made use of the latest versions of the NLTE line formation codes DETAIL and SURFACE (K. Butler, private communication), along with plane-parallel, fully line-blanketed LTE Kurucz atmospheric models.

The physical parameters are estimated using an iterative scheme. The effective temperature is derived from the silicon ionization balance, the gravity from fitting the collisionallybroadened wings of the Balmer lines and the microturbulence from requiring the individual abundances given by the OII lines to be independent of the line strength.

Once the atmospheric parameters above are known, the abundances are derived by matching the observed and predicted equivalent widths of a set of carefully-selected, unblended spectral lines. For consistency, direct integration is used in both cases.

\section{First results}

The derived abundances of two SPBs of early spectral type are given in Table 1. For HD 3360, the abundances of all considered chemical elements are indistinguishable from the values reported for early $B$ dwarfs in the solar neighbourhood within the errors, except for nitrogen. Indeed, we clearly detect a nitrogen excess in this B2 star, as has recently been discovered in four $\beta$ Cephei stars studied by Morel et al. (2006). Similarly to these latter stars, HD 3360 shows a strong boron depletion (Proffitt \& Quigley 2001), has a detected magnetic field and is slowly rotating (Neiner et al. 2003). We refer to Morel et al. (2007) for further discussion on the interpretation of these results. For HD 85953, all derived abundances are solar.

Acknowledgments. MB is a Postdoctoral Fellow of the Fund for Scientific Research, Flanders. We thank all the observers from the Institute of Astrophysics of the University of Leuven who gathered the spectroscopic data. 
Table 1: Atmospheric parameters, NLTE abundances and the resulting metallicity. By convention, $\log \epsilon(\mathrm{H})$ $=12$. The number of used lines is given in brackets. We define $[N / C]$ and $[N / O]$ as $\log [\epsilon(N) / \epsilon(C)]$ and $\log [\epsilon(\mathrm{N}) / \epsilon(\mathrm{O})]$, respectively. For comparison purposes, we also give the typical values found for OB dwarfs in the solar neighbourhood (Daflon \& Cunha 2004), the standard solar composition of Grevesse \& Sauval (1998) and the solar abundances recently derived from 3-D hydrodynamical models (Asplund et al. 2005)

\begin{tabular}{cccccc}
\hline \hline & HD 3360 & HD 85953 & OB stars & Sun 1-D & Sun 3-D \\
\hline$T_{\text {eff }}(\mathrm{K})$ & $22000 \pm 1000$ & $21000 \pm 1000$ & & & \\
$\log g$ & $3.7 \pm 0.15$ & $3.8 \pm 0.15$ & & & \\
$\xi\left(\mathrm{km} \mathrm{s}^{-1}\right)$ & $1 \pm 1$ & $1 \pm 1$ & & & \\
\hline $\mathrm{He} / \mathrm{H}$ & $0.084 \pm 0.027(10)$ & $0.070 \pm 0.012(9)$ & $\sim 0.10$ & $0.085 \pm 0.001$ & $0.085 \pm 0.002$ \\
$\log \epsilon(\mathrm{C})$ & $8.16 \pm 0.08(6)$ & $8.16 \pm 0.14(9)$ & $\sim 8.2$ & $8.52 \pm 0.06$ & $8.39 \pm 0.05$ \\
$\log \epsilon(\mathrm{N})$ & $7.97 \pm 0.13(20)$ & $7.66 \pm 0.20(11)$ & $\sim 7.6$ & $7.92 \pm 0.06$ & $7.78 \pm 0.06$ \\
$\log \epsilon(\mathrm{O})$ & $8.38 \pm 0.30(18)$ & $8.41 \pm 0.32(16)$ & $\sim 8.5$ & $8.83 \pm 0.06$ & $8.66 \pm 0.05$ \\
$\log \epsilon(\mathrm{Mg})$ & $7.57 \pm 0.16(1)$ & $7.62 \pm 0.11(1)$ & $\sim 7.4$ & $7.58 \pm 0.05$ & $7.53 \pm 0.09$ \\
$\log \epsilon(\mathrm{Al})$ & $6.15 \pm 0.16(4)$ & $6.14 \pm 0.13(3)$ & $\sim 6.1$ & $6.47 \pm 0.07$ & $6.37 \pm 0.06$ \\
$\log \epsilon(\mathrm{Si})$ & $7.28 \pm 0.30(6)$ & $7.25 \pm 0.30(6)$ & $\sim 7.2$ & $7.55 \pm 0.05$ & $7.51 \pm 0.04$ \\
$\log \epsilon(\mathrm{S})$ & $7.22 \pm 0.18(7)$ & $7.26 \pm 0.16(6)$ & $\sim 7.2$ & $7.33 \pm 0.11$ & $7.14 \pm 0.05$ \\
$\log \epsilon(\mathrm{Fe})$ & $7.31 \pm 0.16(18)$ & $7.40 \pm 0.23(12)$ & $\sim 7.4$ & $7.50 \pm 0.05$ & $7.45 \pm 0.05$ \\
\hline $\mathrm{Z}$ & $0.010 \pm 0.002$ & $0.010 \pm 0.002$ & $\sim 0.01$ & $0.017 \pm 0.001$ & $0.012 \pm 0.001$ \\
{$[\mathrm{~N} / \mathrm{C}]$} & $-0.19 \pm 0.15$ & $-0.50 \pm 0.24$ & $\sim-0.6$ & $-0.60 \pm 0.08$ & $-0.61 \pm 0.08$ \\
{$[\mathrm{~N} / \mathrm{O}]$} & $-0.41 \pm 0.32$ & $-0.75 \pm 0.37$ & $\sim-0.9$ & $-0.91 \pm 0.08$ & $-0.88 \pm 0.08$ \\
\hline
\end{tabular}

\section{References}

Asplund M., Grevesse N., Sauval A. J., 2005, in Barnes T. G. III, Bash F. N., eds, ASP Conf. Ser. Vol. 336, Cosmic Abundances as Records of Stellar Evolution and Nucleosynthesis. Astron. Soc.

Pac., San Francisco, p. 25

Daflon S., Cunha K., 2004, A\&A, 617, 1115

Grevesse N., Sauval A. J., 1998, Space Sci. Rev., 85, 161

Morel T., Butler K., Aerts C., Neiner C., Briquet M., 2006, A\&A, 457, 651

Morel T., Butler K., Aerts C., Neiner C., Briquet M., 2007, Comm. Asteroseis., 150, 199

Neiner C., Geers V. C., Henrichs H. F., et al., 2003, A\&A, 406, 1019

Proffitt C. R., Quigley M. F., 2001, ApJ, 548, 429

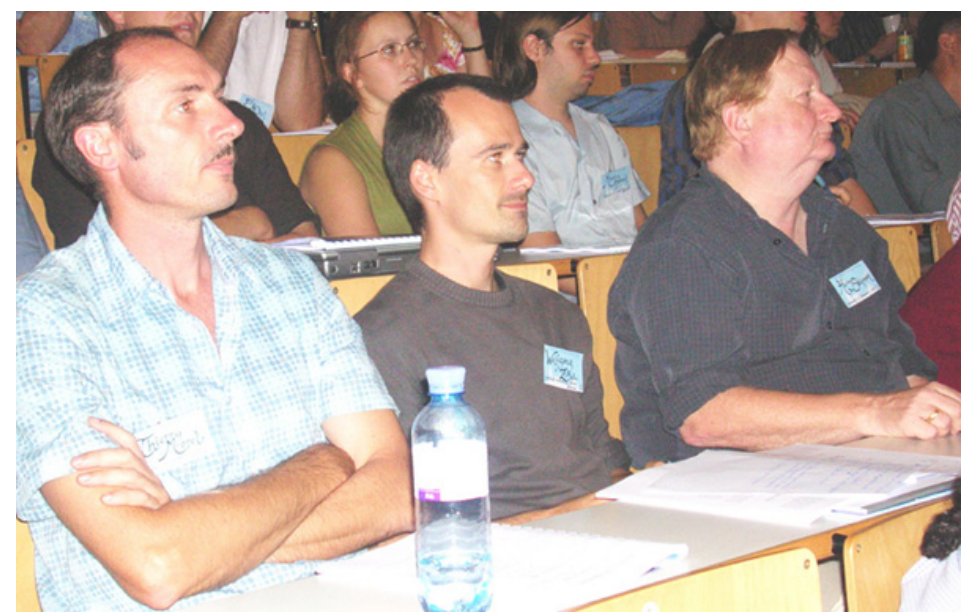

Thierry Morel, Wolfgang Zima and Harry Shipman concentrating on a talk. 